\title{
Developing Active Source Seismology for Planetary Science
}

A white paper submitted to the Planetary Science Decadal Survey 2023-2032

\section{Authors:}

Samuel W. Courville ${ }^{1,2}$

Nathaniel E. Putzig ${ }^{1}$

Matthew R. Perry ${ }^{1,3}$

Gerald W. Patterson ${ }^{4}$

Gareth A. Morgan ${ }^{1}$

Andrew J. Gemer ${ }^{5}$
Paul C. Sava ${ }^{3}$

T. Dylan Mikesell ${ }^{6}$

Richard Degner ${ }^{7}$

Ali M. Bramson ${ }^{8}$

Sean P. S. Gulick ${ }^{9}$

Bjorn Paulsson ${ }^{10}$

\author{
Chance C. Amos ${ }^{1}$ \\ Renee Weber ${ }^{11}$ \\ Mark Panning ${ }^{12}$ \\ Nicholas Schmerr ${ }^{13}$
}

${ }^{1}$ Planetary Science Institute, ${ }^{2}$ Arizona State University, ${ }^{3}$ Colorado School of Mines, ${ }^{4}$ Johns Hopkins University Applied Physics Laboratory, ${ }^{5}$ Lunar Outpost Inc, ${ }^{6}$ Boise State University, ${ }^{7}$ Geophysical Technology, Inc., ${ }^{8}$ Purdue University, ${ }^{9}$ University of Texas at Austin, ${ }^{10}$ Paulsson, Inc., ${ }^{11}$ NASA Marshall Space Flight Center, ${ }^{12}$ California Institute of Technology Jet Propulsion Laboratory, ${ }^{13}$ University of Maryland

\section{Contact information:}

Samuel Courville (swcourville@psi.edu)

Planetary Science Institute

1546 Cole Blvd \#120,

Lakewood, CO 80401

(719)433-8923

\section{Endorsed by:}

Yosio Nakamura (University of Texas at Austin), Bruce Banerdt (California Institute of Technology Jet Propulsion Laboratory), Kris Zacny (Honeybee Robotics), Andrew Jay Feustel (NASA Astronaut Office), Christopher Dreyer, George Sowers, Kevin Cannon (Colorado School of Mines), Jim Bell (Arizona State University), Isaac Smith (York University), Lee Liberty and Zongbo Xu (Boise State University) 


\begin{abstract}
Summary:
Increasingly, the planetary science community has turned toward lander and rover based subsurface investigations to answer science questions about subsurface habitability, geologic history, and the availability of resources like water ice. Yet, as we have advanced many subsurface sounding instruments toward spaceflight readiness, the development of active-source seismology has lagged behind. Despite the success and scientific value of the active-source seismology experiments conducted by Apollo astronauts on the Moon, only small-scale assessments of shallow properties have been carried out since that time, by the hammering of the HP3 probe on Mars next to the InSight seismometers and by the MUPUS experiments on comet 67P/Churyumov-Gerasimenko. No larger-scale planetary active seismology experiments to investigate subsurface properties have been conducted since Apollo. We recommend that the Planetary Decadal Survey place the development and maturation of instrumentation for active-source seismology as a high priority for the coming decade.
\end{abstract}

\section{The Case for Active-Source Planetary Seismology}

We recommend that the Decadal Survey place the development and maturation of activesource seismology as a high priority for the coming decade. Whereas passive seismometers like the Insight Seismic Experiment for Interior Structure (SEIS) are designed to record natural seismic vibrations from earthquakes and meteor impacts, active-source seismology relies on controlled man-made impulsive or vibratory seismic sources in order to survey specific locations. The ability to control the location and frequency content of a seismic source allows for one to target specific subsurface structures. Additionally, a mobile and repeatable active seismic source combined with mobile receivers enables highfidelity 2D and 3D subsurface surveys. This ability is ideal for designing payloads that can meet the vertical and horizontal resolution requirements of mission objectives that require imaging subsurface targets. High priority subsurface targets on the Moon and Mars such as near-surface water ice deposits, habitable subsurface environments, lava tubes, and stratigraphy containing geologic history, could all benefit from active-source seismic surveying.

Key claim 1: Active-source seismology can address key science goals on bodies like the Moon and Mars.
To address many subsurface science exploration goals for the Moon and Mars, the planetary science community has invested in other geophysical sounding instruments like ground penetrating radar (GPR). However, it is important to recognize that one geophysical method does not reign supreme over any other. Different methods are not replacements for each other but rather are highly complementary. For example, whereas seismology measures mechanical properties like compressibility and density, GPR measures electromagnetic properties like permittivity, which may not necessarily be correlated. Depending on the science goals, one method may be more ideal than the other. And together, the two methods provide a more complete picture than either method alone. Separate geophysical methods are complementary, and each brings unique data allowing for multidisciplinary and better informed assessment.

Key claim 2: Active-source seismology is complementary to other subsurface investigation methods such as passive seismology, ground-penetrating radar, and electromagnetic sounding.

Although little planetary active-source seismic exploration has occurred in nearly a halfcentury, the Apollo astronauts demonstrated that the method is an effective tool for characteriz- 
ing the structure of the lunar regolith. Unfortunately, the Apollo active-source seismic experiment relied heavily on the aid of human operation, which greatly hampered broader use of the method in subsequent decades. However, advancements in autonomous technology could now allow robotic active-source seismic systems to be developed in the immediate future.

Key claim 3: Active-source seismic methodology is mature and can be developed for use in planetary science.

The purpose of this white paper is to substantiate Claims $1 \& 2$ by identifying science and human exploration goals where active seismic surveying would be ideal, and Claim 3 by showing that the technology required to conduct a seismic survey robotically exists today, and merely requires maturation for space flight. With modest investment, the development of active seismic systems could be accomplished in the next decade with substantial payoffs in science capabilities.

\section{Active-Source Seismology Science Trace- ability}

Mars Exploration Program Analysis Group (MEPAG) Goal II, Objective B, asks that future explorations "determine the record of the climate of the recent past." To do so will require the characterization of the "locations, composition, and structure of... ice and volatile reservoirs at the surface and near-surface [1]." We now know there is abundant water ice in the shallow subsurface of Mars as equatorward as the mid-latitudes through the discovery of new impact craters which expose ice [2,3], debris covered glaciers which drape massif walls $[4,5,6]$, scarps that expose tens to 100 meters of massive ice [7], widespread subsurface radar interfaces in areas indicative of ice-rich terrains [8,9], inferred water contents from neutron spectroscopy [10], and thermal signatures [11]. However, Bramson et al. [12] (WP2020) explain that the depth to the top of the ice, and properties such as ice concentration and heterogeneity remain elusive to observe from current orbital datasets. Terrestrial studies of permafrost regions have demonstrated that the percentage of ice within the ground has a measurable effect on the seismic velocity of the material $[13,14]$. Thus, an active-source seismic system landed on Mars could determine the depth to, concentration, and heterogeneity of water ice deposits.

The current Planetary Science Decadal Survey, Visions and Voyages [15], set characterization of the lunar volatile cycle as a top priority for lunar exploration, seeking to address if and how volatiles migrate across the lunar surface, and what is their ultimate fate. In the last two decades, much has been uncovered about the presence of water ice in lunar regolith. Neutron data indicates that up to a few volume percent of water ice could be present within some polar craters [16, 17]. The LCROSS impact into the lunar south pole revealed water ice between 3 and $10 \%$ by weight from the vapor in the resulting ejecta plume [18, 19]. Furthermore, a diverse set of observations from the NASA Lunar Reconnaissance Orbiter (LRO) suggests that the Moon possesses reservoirs of water/waterice concentrated in the polar regions $[20,21,22$, $23,24,25]$. These data suggest water is heterogeneously distributed on multiple scales within the top meter of the lunar polar regolith. However, thick ice deposits on the Moon have yet to be confirmed [26, 27], and the need for surface exploration of Lunar ice deposits is expressed in Hayne et al. [28] (WP2020). The ambiguity associated with detecting subsurface ice from orbit and beyond strongly argues for the need for landed missions within permanently shadowed regions. Active-source seismology is a promising method because the increased seismic velocity induced by the presence of ice within the regolith $[13,14]$ provides a means to constrain ice content. Additionally, abrupt transitions in ice content, such as the top and bottom of an ice deposit, would produce strong seismic-wave reflections and refractions. 
Key finding: Active-source seismology could identify subsurface water ice, a key to climate and formation history

Under MEPAG Goal III, Objective A2 suggests that future investigations should "document the geologic record preserved in sediments and sedimentary deposits," and "constrain the location, volume, timing, and duration of past hydrologic cycles that contributed to the sedimentary and geomorphic record." Additionally, Objective A4 says to "determine the nature and timing of construction and modification of the crust," and "determine the absolute and relative ages of geologic units and events through martian history." For locations where martian stratigraphy is not readily exposed at the surface, the ideal method to reveal the chronology of layers, as demonstrated by extensive application on earth, is active-source seismology.

Lava tubes are also a geologic target of interest, yet their detection from orbit is controversial $[29,30]$ (WP2020). Orbital imagery suggest that large lava tubes exist on both the Moon and Mars, yet confirmation from orbit has remained elusive. Lava tubes represent both a record of volcanic geologic history, and potentially habitable locations shielded from harmful solar radiation. Since lava tubes could be large and deep

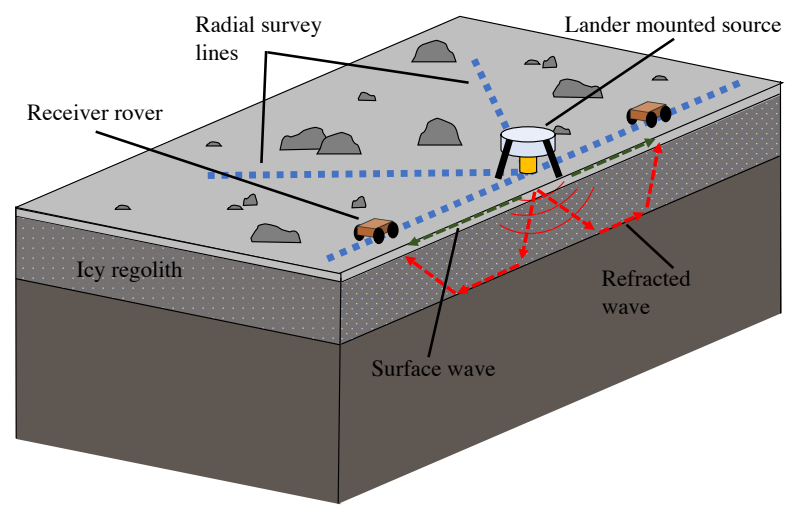

(a) An active seismic refraction and surface wave experiment using a lander mounted seismic source on the Moon and Mars, seismology may be a better method of detection and delineation than electromagnetic methods.

Key finding: Active-source seismology can investigate subsurface geology, like sedimentary stratigraphy and lava tubes, which unlock hidden geologic histories.

We do not suggest that active seismology is the sole method to accomplish these goals, but in some cases, it may be the ideal method. Regardless, every geophysical method has limitations. One method cannot answer every question about the subsurface. For instance, ground penetrating radar may not be able to distinguish ice from highly porous material, because both features would have low permittivity. On the other hand, water ice has a high seismic velocity, whereas porous material has a low seismic velocity, allowing the two to be distinguished via activesource seismology. Morgan et al. [31] demonstrate through orbital mapping of ice deposits on Mars that combining multiple geophysical data sets yields more accurate knowledge of the subsurface than any one dataset alone.

Key finding: Active-source seismology is complementary to other subsurface investigation methods.

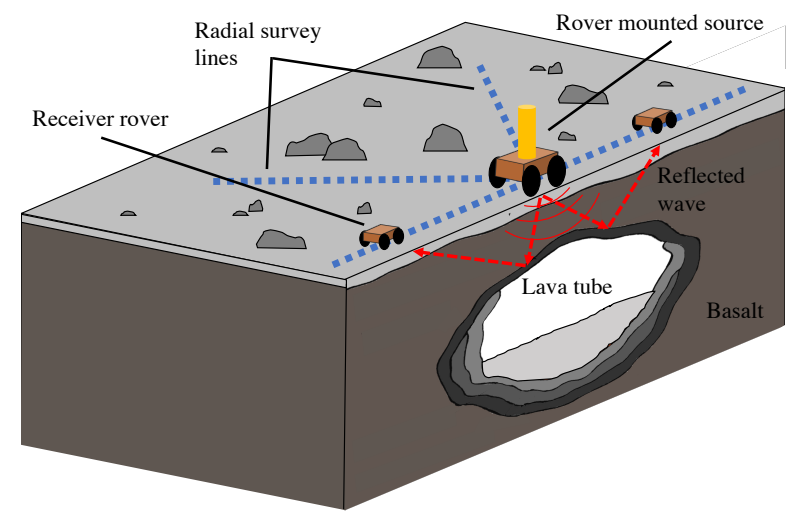

(b) An active seismic reflection experiment using a seismic source mounted on a rover

Figure 1: Concepts for how active seismology could be applied on a planetary body. 


\section{Active-Source Seismology's Benefit to Fu- ture Human Exploration}

In advance of human exploration of Mars, and renewed sustainable human exploration of the Moon, the delineation of proven reserves of water ice will be necessary to enable in-situ resource utilization (ISRU) which would provide life support and propellant [32]. In combination with ground-truth sampling, active-source seismology could survey the depth and extent of subsurface water ice over a large landing site area. Under MEPAG Goal IV, Objective $\mathrm{C} 2$ suggests that the community must "characterize potentially extractable water resources to support ISRU for long-term human needs," and to "prepare high spatial resolution maps of one equatorial site with water bound in regolith materials and one mid to high latitude site with water ice at or within a few meters of the surface...."Active-source seismology would be an excellent method for landing site resource characterization because it can allow for full 3D imaging over a sufficiently large area, an improvement over limited discrete sampling or 1D sounding.

Within the exploration objectives of NASA's Human Exploration and Operations Mission Directorate [33], the phase 1 objective P1-17 states the need to "evaluate the nature and distribution of lunar volatiles...to inform future ISRU development." Whether or not human exploration of the Moon can rely on water ice deposits in permanently shadowed regions strongly depends on the distribution and concentration of the water ice. Active-source seismology could help survey a potential landing site for water ice and thus help answer a vital HEOMD question: can water ice be practically used during human exploration of the Moon?

Key finding: Active-source seismology is a valuable tool for site characterization and resource surveying in advance of human exploration.

\section{Implementation Strategy and Required Technology}

On Earth, active-source seismology is the most well developed and widely used method for subsurface surveying and resource exploration. Whereas instrumentation is mass produced for terrestrial applications, insufficient space-ready instrumentation exists for planetary science application today. In the past, active seismic surveying proved to be a valuable method to reveal regolith structure during the Apollo missions [34, 35], but these surveys required assistance from astronauts. Thus the lack of recent active seismic experiments could be attributed to the limitations of robotic capabilities and autonomous navigation, but such challenges are now surmountable. An active-source seismology system requires two instruments, a seismic source generator, and a receiver (e.g. a geophone or seismometer). To conduct a full seismic survey over a subsurface target region, there must either be multiple sources and receivers spaced over the area, or mobile sources and receivers that traverse over the survey area. The following paragraphs briefly introduce potential seismic source and receiver instruments that could be developed for planetary science.

Seismic sources: Accelerated weight-drop systems are repeatable impulsive sources that are simple, robust, and commercially available, however they have not yet been applied in space. Modern accelerated weight-drop systems use hydraulic arms to lift a source mass and subsequently accelerate it toward the surface, generating a seismic pulse upon colliding with the ground. These sources can operate on city streets with no surface damage and are scalable to image the near surface or depths exceeding one kilometer [36, 37]. The amplitude of the excited seismic waves, and thus the maximum distance that a seismic wave can travel and still be detectable, is largely dependent on the kinetic energy of the weight drop and the peak frequency of the resulting source pulse. Depending on the desired survey area and depth, 
an accelerated weight-drop source could weigh 10s-100s of kilograms, and could be mounted on a large lander or rover. Similar to accelerated weight drop sources are vibroseis sources that press a weight against the ground and vibrate it with specific frequency pattern. Such machines are mechanically complex, but achieve greater survey control and higher signal to noise ratios. Other seismic sources include explosives, such as dynamite, standard initiators (small explosives used to deploy solar panels, also used as sources for the Apollo ASE), and seisguns or mortars (projectiles accelerated with gunpowder or explosives). Explosive sources could deliver the same energy as a weight-drop system with a fraction of the mass, however, they are not repeatable.

Seismic receivers: The standard seismic receiver is a geophone. Geophones differ from passive seismometers mainly in the frequency of waves they record; geophones record vibration frequencies in the $10 \mathrm{~s}-100 \mathrm{~s} \mathrm{~Hz}$ range whereas seismometers must record waves as low as 0.01 $\mathrm{Hz}$ which requires extreme sensitivity. Terrestrial geophones that record vibrations in three orthogonal directions often weigh less than $1 \mathrm{~kg}$, and are cheap to mass produce. Geophones that wirelessly transmit their data, like GTI's nodal geophones [38], could be deployed or incorporated into a mobile rover without any of the cables or wires typically seen in terrestrial seismic surveys. In addition to conventional geophones, Microelectromechanical systems (MEMS), the small accelerometers within smartphones, are nearing the sensitivity required for seismic exploration. Their extremely light weight, low energy usage, and durability are major advantages for space applications. Another potential receiver is fiber optic sensing [39]. Fiber optic geophones function by sending pulses of light into a fiber optic cable that is coupled to the ground and subsequently recording the light that scatters back, which is sensitive to minute strains in the cable, such as those caused by seismic ground motion. These sensors have been deployed in terrestrial seismic experiments with promising results [40]. Lastly, Sava and Asphaug [41] and Courville and Sava [42] propose using non-contact laser Doppler vibrometers (LDV) to record seismic signals. LDVs measure ground vibrations remotely by observing changes to the frequency of a laser beam reflected off of the ground surface, which is similar to how police radar speed guns work. Such an instrument could be operated from an aerial or orbital craft, and would be ideal for a seismic survey on an asteroid or comet. In this scenario, it would have numerous technical advantages over a landed geophone because it would not need complicated mechanical components and because it could characterize ground oscillations at many distributed locations by simply pointing.

Key finding: Many seismic source and receiver instrument options exist for terrestrial applications, and their development for application in space would enable a wide variety of planetary active seismic mission concepts.

In order to conduct a planetary active-source seismology survey, the source and receiver components must be put together into a complete payload concept. One such concept, the Autonomous Roving Exploration System (ARES), could conduct an active-source seismic survey on the Moon or Mars over an area of up to a square kilometer, and see to depths of up to a kilometer [43]. ARES requires multiple rovers: one large rover or lander would include a weight-drop seismic source instrument, and one or more small rovers would contain seismic receivers. As depicted in Figure 1, the system acquires data by generating a seismic source and recording surface waves, refractions, and reflections from subsurface structure at various locations offset from the source. A collection of autonomous receiver rovers provide configurability and redundancy to collect enough data 
to survey a landing site. The receiver rover(s) can be compact and low-mass, allowing multiple rovers to be deployed on each mission and maximizing survey area. An example option is the $10 \mathrm{~kg}$ Mobile Autonomous Prospecting Platform (MAPP) rover produced by Lunar Outpost Inc, which would navigate autonomously between survey points and traverse up to $8 \mathrm{~km}$ in its lifetime [44]. The weight drop source instrument could be accommodated by a moderate or large sized rover/lander such as a Phoenix class lander, a commercial lunar payload system, a spirit/opportunity class rover, or Lunar outpost's $300 \mathrm{~kg}$ HL-MAPP rover.

Larger and smaller scale active seismic experiments can also be conceptualized. For larger scale seismic surveys that must image deeper than a few kilometers, large seismic receiver arrays, such as those described in Fouch et al. [45], would be possible with the aid of large explosive or impact sources and rovers that could travel long distances to deploy receivers at a significant spread. Smaller scale active-source seismic characterization on the order of meters or less could be easily conducted through the wheels or legs of a rover or lander using small accelerometers as seismic receivers. Such an experiment has already been deployed on comet 67P/Churyumov-Gerasimenko. [46]

Key finding: Instruments and payload concepts exist to operate active-source seismology instrumentation on the surface of the Moon, Mars, and beyond.

Seismic data processing techniques are highly matured. For near surface investigation (0-10m), seismic surface-wave and refraction analysis is a standard method on Earth to measure variations of near-surface composition and layering with depth. Specifically, multi-channel analysis of surface waves can reveal the seismic velocity profile with depth [47]. For deeper targets (10s-1000s of meters), seismic wavefield migration and tomography are well developed.
Migration [48, 49] is designed to infer interface properties, i.e. the contrast of physical properties at all locations in the investigated volume, while tomography $[50,51]$ is designed to infer volumetric properties, e.g. the seismic velocity at every in the volume. Both techniques are well developed in exploration seismology and are complementary to one-another, i.e. information from one improves the effectiveness of the other. Full waveform inversion methodologies that incorporate both amplitude and phase of the seismic signals are now available and provide both $3-\mathrm{D}$ velocity variations and layering structure [52].

Key finding: Seismic surveying and data processing methodology are well matured from extensive terrestrial application.

\section{Benefits to Space Technology Develop- ment}

Active-source seismology would provide an excellent test bed for technology demonstrations. Active-source seismology relies on the ability to separate a source and receiver in space, and to record data with the receiver placed at different offsets from the source. Since an active-source seismic system might require two or more separate rovers working together over a survey region, implementing an activesource seismic system would be an excellent platform to demonstrate autonomously navigating rovers that communicate and work together to survey a region with applications beyond active-source seismic acquisition. For example, ground-penetrating radar or sample collection could be employed in tandem with a seismic system.

Key finding: Active-source seismology provides a test bed for autonomous exploration systems, which would be a major technological demonstration for the future of planetary science exploration. 


\section{Conclusion}

We recommend that the NRC place strong emphasis on developing active-source seismic instrumentation for planetary exploration in the coming decade. The lack of seismology instrumentation for planets is a travesty for planetary exploration, and limits subsurface planetary investigation.

\section{References}

[1] V. Hamilton et al. MEPAG, 2015. [2] S. Byrne et al. Science, 325:1674-1976, 2009. [3] C. M. Dundas et al. J. Geophys. Res. Planets, 119:109-127, 2014. [4] J. W. Holt et al. Science, 322: 1235-1238, 2008. [5] J. J. Plaut et al. Geophys. Res. Lett., 36(L02203):1-4, 2009. [6] E. I. Petersen et al. Geophys. Res. Lett., 45(21):11,595-11,604, 2018. [7] C. M. Dundas et al. Science, 359(6372): 199-201, 2018. [8] A. M. Bramson et al. Geophys. Res. Lett., 42(16):6566-6574, 2015. [9] C. M. Stuurman et al. Geophys. Res. Lett., 43(18):9484-9491, 2016. [10] A. V. Pathare et al. Icarus, 301: 97-116, 2018. [11] S. Piqueux et al. Geophys. Res. Lett., 46(3):1-9, 2019. [12] A. M. Bramson et al. Mid-latitude ice on Mars: A science target for planetary climate histories and an exploration target for in situ resources. White paper, 2020. [13] R. Zimmerman and M. King. Geophys., 51(6):1285-1290, 1986. [14] T. Johansen et al. Geophys., 68(2):566-573, 2003. [15] National Research Council. Vision and Voyages for Planetary Science in the Decade 2013-2022. The National Academies Press,

Washington, DC, 2011. ISBN 978-0-309-22464-2. doi: 10.17226/13117. [16] W. C. Feldman et al. Science, 281:1496-1500, 1998. [17] A. B. Sanin et al. Icarus, 2016. [18] A. Colaprete et al. Science, 330:463-468, 2010. [19] J. L. Heldmann et al. Icarus, 254:262-275, 2015. [20] Mitrofanov et al. J. Geophys. Res., 117, 2012. [21] P. D. Spudis et al. J. Geophys. Res., 118:2016-2029, 2013. [22] P. G. Lucey et al. J. Geophys. Res., 119:1665-1679, 2014. [23] Hayne et al. Icarus, 255:58-69, 2015. [24] McClanahan et al. Icarus, 255:88-99, 2015. [25] Patterson et al. Icarus, 283:2-19, 2017. [26] L. Rubanenko et al. Nature, 12:597-601, 2019. [27] A. Deutsch et al. Icarus, 336:113455, 2020. [28] P. O. Hayne et al. New approaches to lunar ice detection and mapping. White paper, 2020. [29] T. N. Titus et al. Science and technology requirements to explore caves in our solar system. White paper, 2020. [30] J. G. Blank et al. Volcanic caves as priority sites for astrobiology science. White paper, 2020. [31] G. A. Morgan et al. Nature, in review, 2020. [32] A. Abbud-Madrid et al. Report of the Mars Water In-Situ Resource Utilization (ISRU) Planning (M-WIP) Study, 90, 2016. [33] HEOMD. Human exploration and operations exploration objectives. NASA, REVISION A, 2017. [34] R. Kovach and J. Watkins. The moon, 7(1):63-75, 1973. [35] G. Dal Moro. Icarus, 254: 338-349, 2015. [36] L. M. Liberty et al. Bulletin of the Seismological Society of America, 98(4):1681, 2008. [37] L. M. Liberty. The Leading Edge, 30(2):936-941, 2011. [38] Geophysical Technology Inc. URL https: / / geophysicaltechnology.com/. [39] C. C. Amos et al. 50th Lunar and Planetary Science Conference, 2623, 2019. [40] Tom Parker et al. first break, 32(2), 2014. [41] Paul Sava and Erik Asphaug. Advances in Space Research, 64(2):527-544, 2019. [42] Samuel W Courville and Paul Sava. Acta Astronautica, 172:16-32, 2020. [43] S. Courville et al. AGU Fall Meeting, P54D-02, 2018. [44] Lunar Outpost. URL https : / / www . lunaroutpost. com/. [45] M.J. Fouch et al. 40th Lunar and Planetary Science Conference, 2233, 2009. [46] M. Knapmeyer et al. Icarus, 310:165 - 193, 2018. [47] C. B. Park et al. Geophysics, 64(3):800-808, 1999. [48] A. J. Berkhout. Imaging of Acoustic Energy by Wave Field Extrapolation. Elsevier, 1982. [49] J. F. Claerbout. Imaging the Earth's Interior. Blackwell Scientific Publications, 1985. [50] A. Tarantola. Inverse Problem Theory: Methods for Data Fitting and Model Parameter estimation. Elsevier, 1987. [51] G. Nolet. A Breviary of Seismic Tomography: Imaging the Interior of the Earth and Sun. Cambridge University Press, 2008. [52] D. Vigh and E. W. Starr. Geophysics, 73:135-144, 2008. 